\title{
Structural analysis of segmented tunnel rings: A combination of transfer relations with an interface law
}

\author{
J.L. Zhang \& H.A. Mang \\ Institute for Mechanics of Materials and Structures, TU Wien-Vienna University of Technology, \\ Vienna, Austria \\ College of Civil Engineering, Tongji University, Shanghai, China
}

X. Liu \& Y. Yuan

College of Civil Engineering, Tongji University, Shanghai, China

B. Pichler

Institute for Mechanics of Materials and Structures, TU Wien -Vienna University of Technology, Vienna, Austria

\begin{abstract}
Structural analysis of segmented tunnel rings is challenging, because displacement discontinuities and relative rotation angles develop at segment-to-segment interfaces. To meet this challenge, a hybrid method was recently developed by Zhang et al. (2017). It uses the known external loading and measured interfacial discontinuities as input for structural analysis. The latter is based on analytical transfer relations, representing solutions of the governing equations of the linear theory of slender circular arches. The present contribution goes one step further. In order to enable structural analysis without measured interfacial discontinuities, the transfer relations are combined with an interface law. The latter represents a mathematical relation between the bending moment transmitted across a typical segment-to-segment interface and the resulting relative rotation angle. The proposed approach is applied to linear structural analysis of the first four load steps of a real-scale test on a segmented tunnel ring. Corresponding results are compared with the output of the aforementioned hybrid method. This way, it is shown that the presented method delivers reasonable estimates of the inner forces. However, the displacements are predicted less accurately, because rigid body motions of the segments cannot be quantified by interface laws.
\end{abstract}

\section{INTRODUCTION}

In hybrid methods, monitored data are used as input for structural simulations. Concerning the analysis of tunnel linings, Rokahr (Rokahr and Zachow 1997) and Hellmich (Hellmich 1999, Hellmich et al. 1999, Hellmich et al. 2001) developed the first hybrid methods for the New Austrian Tunneling Method. They are nowadays frequently applied in construction of tunnels (Brandtner et al. 2007), in order to quantify stress levels in shotcrete tunnel shells on the basis of measured displacements. Follow-up research activities were concerned with the development of modern multiscale material models for hydrating shotcrete (Pichler et al. 2008). This allowed for (i) reducing the required experimental activities to calorimetry testing of the binder of the shotcrete (Ullah et al. 2012), (ii) assessing the robustness of safety analyses of shotcrete tunnel shells with respect to reboundrelated uncertainties of the effective composition of shotcrete, finally arriving at the tunnel wall (Ullah et al. 2012), and for (iii) identifying a non-trivial mode of arching thrustline behavior of young topheading shotcrete tunnel shells, subjected to both ground pressure and ground shear (Ullah et al. 2013).

As for mechanized tunneling, the first hybrid method was recently presented by Zhang et al. (2017). The authors re-analyzed the first four load steps of a real-scale laboratory test on a segmented tunnel ring. The structure was subjected to 24 hydraulic jack forces, simulating the ground pressure. In the experiment, the displacement discontinuities and the relative rotation angles at all six segment-to-segment interfaces were monitored (Liu et al. 2016). These measurements were used as input for hybrid analysis of the structural behavior of the tested tunnel ring (Zhang et al. 2017). During regular service of segmented tunnel linings, however, interfacial discontinuities of displacements and rotation angles are unknown. This provides the motivation to apply interface laws to structural analysis of segmented tunnel rings. 


$$
\left[\begin{array}{c}
u(\varphi) \\
v(\varphi) \\
\theta(\varphi) \\
M(\varphi) \\
N(\varphi) \\
V(\varphi) \\
1
\end{array}\right]=\left[\begin{array}{ccccccc}
\cos \varphi & \sin \varphi & T_{13}(\varphi) & T_{14}(\varphi) & T_{15}(\varphi) & T_{16}(\varphi) & \sum u^{L}(\varphi) \\
-\sin \varphi & \cos \varphi & T_{23}(\varphi) & T_{24}(\varphi) & T_{25}(\varphi) & T_{26}(\varphi) & \sum v^{L}(\varphi) \\
0 & 0 & 1 & T_{34}(\varphi) & T_{35}(\varphi) & T_{36}(\varphi) & \sum \theta^{L}(\varphi) \\
0 & 0 & 0 & 1 & T_{45}(\varphi) & T_{46}(\varphi) & \sum M^{L}(\varphi) \\
0 & 0 & 0 & 0 & \cos \varphi & -\sin \varphi & \sum N^{L}(\varphi) \\
0 & 0 & 0 & 0 & \sin \varphi & \cos \varphi & \sum V^{L}(\varphi) \\
0 & 0 & 0 & 0 & 0 & 0 & 1
\end{array}\right] \cdot\left[\begin{array}{c}
u_{i} \\
v_{i} \\
\theta_{i} \\
M_{i} \\
N_{i} \\
V_{i} \\
1
\end{array}\right] .
$$

Interface laws are mathematical functions relating the originally unknown relative rotation angles at segment-to-segment interfaces to the normal force and the bending moment transmitted across these interfaces. Popular interface laws were developed by Leonhardt and Reimann (1965), Gladwell (1980), and Janßen (1983). As for the special case of interfaces subjected to eccentric compression, these state-of-the-art interface laws suggest that relative rotation angles increase linearly with increasing loading (Schlappal et al. 2017). This allows for a reliable description of Serviceability Limit States, but does not account for the nonlinearities associated with Ultimate Limit States concerning the bearing capacity of the interfaces (Schlappal et al. 2017). As regards the desirable development of nonlinear interface laws, only first steps are documented in the open literature, see e.g. (Liu et al. 2017) and references therein. Generally applicable models are still lacking.

In this paper, a linear interface law taken from (Liu et al. 2017) is combined with transfer relations representing analytical solutions of the governing equations of the linear theory of slender circular arches (Zhang et al. 2017). Because of the linear nature of the performed structural analyses, the present focus is resting on segmented tunnel rings subjected to external loads which are significantly smaller than the bearing capacity. This was the motivation to restrict the analyses to the first four load steps of the aforementioned realscale laboratory test on a segmented tunnel ring. Notably, the segments showed no sign of bendinginduced tensile cracking during the analyzed initial phase of the test (Liu et al. 2016). Therefore, linear elastic behavior of the reinforced concrete segments and a linear interface law are reasonable assumptions.

The experimentally applied hydraulic jack forces enter the structural analysis as input, while the interfacial discontinuities are predicted by the interface law. This results in classical load-controlled simulations. Corresponding numerical results are compared with available results from the hybrid method. This allows for assessing both qualitatively and quantitatively to which extent a classical structural analysis, i.e. one without use of measured displacements, can provide insight into the structural behavior of a segmented tunnel ring.

The paper is structured as follows. Section 2 refers to analytical solutions for segmented tunnel rings. The transfer relations for such structures and the proposed interface law are briefly summarized. In addition, analytical solutions for integration constants and relative rotation angles are derived. In Section 3, the proposed mode of analysis is applied to the first four load steps of a real-scale test on a segmented tunnel ring. Section 4 contains the conclusions drawn from the present research.

\section{ANALYTICAL SOLUTIONS FOR SEGMENTED TUNNEL RINGS}

\subsection{Transfer relations (Zhang et al. 2017)}

In this paper, structural analysis of segmented tunnel rings is based on transfer relations, representing analytical solutions of the governing equations of the linear theory of slender circular arches (Zhang et al. 2017). Eq. (1), see above, contains the matrixvector representation of the transfer relations, following the notation used by Rubin and Vogel (1993). The components $T_{13}$ to $T_{46}$ of the transfer matrix read as (Zhang et al. 2017)

$$
\begin{aligned}
T_{13}(\varphi) & =R \sin \varphi, \\
T_{14}(\varphi) & =\frac{R^{2}}{E I}(\cos \varphi-1), \\
T_{15}(\varphi) & =\frac{R}{E A} \frac{1}{2} \varphi \sin \varphi \\
& +\frac{R^{3}}{E I}\left(\frac{1}{2} \varphi \sin \varphi+\cos \varphi-1\right),
\end{aligned}
$$




$$
\begin{aligned}
T_{16}(\varphi) & =\frac{R}{E A}\left(\frac{1}{2} \varphi \cos \varphi-\frac{1}{2} \sin \varphi\right) \\
& +\frac{R^{3}}{E I}\left(\frac{1}{2} \varphi \cos \varphi+\frac{1}{2} \sin \varphi\right)
\end{aligned}
$$$$
T_{23}(\varphi)=R(\cos \varphi-1)
$$$$
T_{24}(\varphi)=\frac{R^{2}}{E I}(\varphi-\sin \varphi),
$$$$
T_{25}(\varphi)=\frac{R}{E A}\left(\frac{1}{2} \varphi \cos \varphi+\frac{1}{2} \sin \varphi\right)
$$$$
+\frac{R^{3}}{E I}\left(\varphi-\frac{3}{2} \sin \varphi+\frac{1}{2} \varphi \cos \varphi\right),
$$$$
T_{26}(\varphi)=\frac{R}{E A}\left(-\frac{1}{2} \varphi \sin \varphi\right)
$$$$
+\frac{R^{3}}{E I}\left(1-\cos \varphi-\frac{1}{2} \varphi \sin \varphi\right),
$$$$
T_{34}(\varphi)=-\frac{R}{E I} \varphi,
$$$$
T_{35}(\varphi)=\frac{R^{2}}{E I}(\sin \varphi-\varphi),
$$$$
T_{36}(\varphi)=\frac{R^{2}}{E I}(\cos \varphi-1),
$$$$
T_{45}(\varphi)=R(1-\cos \varphi),
$$$$
T_{46}(\varphi)=R \sin \varphi
$$

In Eqs. (1)-(14), $R, E A$, and $E I$ denote the radius of the neutral axis of the arch, the extensional stiffness, and the bending stiffness, respectively. The vector on the left-hand-side of Eq. (1) contains the kinematic and static variables, referring to the cross-section at an arbitrary value of the angular coordinate $\varphi$, i.e. the radial displacement $u$, the circumferential displacement $v$, the cross-sectional rotation $\theta$, the bending moment $M$, the normal force $N$, and the shear force $V$. The summation symbols in the last column of the transfer matrix in Eq. (1) refer to the superposition of so-called "load integrals". The latter represent analytical solutions for (i) radial point loads and (ii) relative rotation angles at segment-tosegment interfaces. The load integrals for a radial point load $P$, acting at position $\varphi_{p}$, read as (Zhang et al. 2017):

$$
\begin{aligned}
u_{P}^{L}(\varphi)= & \frac{1}{2}\left(\frac{P R}{E A}+\frac{P R^{3}}{E I}\right)\left[\left(\varphi-\varphi_{p}\right) \cos \left(\varphi-\varphi_{p}\right)\right. \\
& \left.-\sin \left(\varphi-\varphi_{p}\right)\right] H\left(\varphi-\varphi_{p}\right), \\
v_{P}^{L}(\varphi)= & \left\{-\frac{P R}{E A}\left[\frac{1}{2}\left(\varphi-\varphi_{p}\right) \sin \left(\varphi-\varphi_{p}\right)\right]\right. \\
& -\frac{P R^{3}}{E I}\left[\frac{1}{2}\left(\varphi-\varphi_{p}\right) \sin \left(\varphi-\varphi_{p}\right)\right. \\
& \left.\left.+\cos \left(\varphi-\varphi_{p}\right)-1\right]\right\} \quad H\left(\varphi-\varphi_{p}\right), \\
\theta_{P}^{L}(\varphi)= & \frac{P R^{2}}{E I}\left[\cos \left(\varphi-\varphi_{p}\right)-1\right] H\left(\varphi-\varphi_{p}\right) \\
M_{P}^{L}(\varphi)= & R P \sin \left(\varphi-\varphi_{p}\right) H\left(\varphi-\varphi_{p}\right), \\
N_{P}^{L}(\varphi)= & -P \sin \left(\varphi-\varphi_{p}\right) H\left(\varphi-\varphi_{p}\right), \\
V_{P}^{L}(\varphi)= & P \cos \left(\varphi-\varphi_{p}\right) H\left(\varphi-\varphi_{p}\right),
\end{aligned}
$$

where $H\left(\varphi-\varphi_{p}\right)$ stands for the Heaviside function. The load integrals for a relative rotation angle, $\Delta \theta_{j}$, across an interface positioned at the angular coordinate $\varphi_{j}$, read as (Zhang et al. 2017):

$$
\begin{aligned}
& u_{\Delta \theta}^{L}(\varphi)=-R \Delta \theta_{j} \sin \left(\varphi-\varphi_{j}\right) H\left(\varphi-\varphi_{j}\right), \\
& v_{\Delta \theta}^{L}(\varphi)=R \Delta \theta_{j}\left[1-\cos \left(\varphi-\varphi_{j}\right)\right] H\left(\varphi-\varphi_{j}\right), \\
& \theta_{\Delta \theta}^{L}(\varphi)=\Delta \theta_{j} H\left(\varphi-\varphi_{j}\right), \\
& M_{\Delta \theta}^{L}(\varphi)=N_{\Delta \theta}^{L}(\varphi)=V_{\Delta \theta}^{L}(\varphi)=0 .
\end{aligned}
$$

The state vector on the right-hand-side of Eq. (1) refers to the initial cross-section, marked by the index " $i$ ". The corresponding kinematic and static variables, $u_{i}, v_{i}, \theta_{i}, M_{i}, N_{i}$, and $V_{i}$, represent unknown integration constants (Zhang et al. 2017). Further unknowns are the relative rotation angles at the interfaces. They intervene in the interface law, introduced next.

\subsection{Linear interface law}

In the present contribution, a simple interface law is used for the description of the six segment-tosegment interfaces of a segmented tunnel ring. The interface law relates the bending moments transmitted across the six interfaces, $M_{i}, j=1,2, \ldots, 6$, to the resulting relative rotation angles of the interfaces, $\Delta \theta_{j}, j=1,2, \ldots, 6$, using a rotational spring stiffness as the proportionality factor. In this context, it is emphasized that the analyzed segment-tosegment interfaces is unsymmetric with respect to the horizontal middle axis of the cross-section of the segment. This is the consequence of eccentric 
bolts, used to connect neighboring segments. Therefore, the effective rotational spring stiffnesses of the interfaces depends on the orientation of the bending moments transmitted across the interfaces. The designations $K^{+}$and $K^{-}$are used for a positive and a negative bending moment, respectively. Thus, the interface law reads as

$$
\left.\begin{array}{l}
M_{j}=K^{+} \Delta \theta_{j}, \text { if } \quad M_{j}>0 \\
M_{j}=K^{-} \Delta \theta_{j}, \text { if } \quad M_{j}<0
\end{array}\right\} j=1,2, \ldots, 6 .
$$

\subsection{Derivation of a linear system of equations allowing for the analytical calculation of the integration constants and the relative rotation angles}

Identification of the six integration constants $u_{i}, v_{i}$, $\theta_{i}, M_{i}, N_{i}$, and $V_{i}$ and of the six relative rotation angles at the six interfaces of a segmented tunnel ring requires

$$
\left[\begin{array}{l}
M_{i} \\
N_{i} \\
V_{i} \\
\Delta \theta_{1} \\
\Delta \theta_{2} \\
\vdots \\
\Delta \theta_{6}
\end{array}\right]=-\left[\begin{array}{cccc}
0 & 0 & \frac{R \pi+R^{3} \pi}{E A} E I & -R \sin \varphi_{1} \\
\frac{2 R^{2} \pi}{E I} & \frac{R \pi R^{3} \pi}{E A E I} & 0 & R\left(1-\cos \varphi_{1}\right) \\
-\frac{2 R \pi}{E I} & -\frac{2 R^{2} \pi}{E I} & 0 & 1 \\
1 & R\left(1-\cos \varphi_{1}\right) & R \sin \varphi_{1} & K_{1} \\
1 & R\left(1-\cos \varphi_{2}\right) & R \sin \varphi_{2} & 0 \\
\vdots & \vdots & \vdots & \vdots \\
1 & R\left(1-\cos \varphi_{6}\right) & R \sin \varphi_{6} & 0
\end{array}\right.
$$

twelve equations. Notably, the kinematic variables $u_{i}, v_{i}$, and $\theta_{i}$ refer to rigid body motions of the segmented tunnel ring. Without loss of generality, they may be set equal to zero, i.e.

$$
u_{i}=v_{i}=\theta_{i}=0 \text {, }
$$

reducing the number of required equations to nine.

As for the identification of the three static variables $M_{i}, N_{i}$, and $V_{i}$, three geometric continuity conditions are formulated for the closed segmented tunnel ring (Zhang et al. 2017). To this end, the transfer relations are specified for $\varphi=2 \pi$, resulting in a relation between the state variables at the initial and the final (index " $f$ ") cross-section. Since the final cross-section is equal to the initial crosssection, the geometric compatibility conditions are obtained as $u_{f}=u_{i}, v_{f}=v_{i}$, and $\theta_{f}=\theta_{i}$. This delivers three linear equations for the nine unknowns.
The remaining six equations are obtained from formulating the interface law (25) for all six interfaces. To this end, the fourth line of the transfer relations (1) is specified for the position of the interfaces, i.e. for $\varphi=\varphi_{i}, j=1,2, \ldots, 6$. The obtained expression for the six bending moments $M_{i}, j=1,2, \ldots, 6$, are inserted into the interface law (25). This delivers additional six linear equations for the nine unknowns.

The described nine linear equations can be solved analytically, see Eq. (47). Therein, $K_{1}, K_{2}$, ..., $K_{6}$ stand for the rotational spring stiffnesses of the six interfaces. They are either equal to $K^{+}$or to $K^{-}$, depending on the sign of the corresponding bending moments $M_{1}, M_{2}, \ldots, M_{6}$, see Eq. (25). Notably, these signs are originally unknown.

\subsection{Solution strategy for the system of equations (26)}

Generally, a typical trial-and-error approach is required for solving the system of equations (26).

$\left.\begin{array}{ccc}-R \sin \varphi_{2} & \cdots & -R \sin \varphi_{6} \\ R\left(1-\cos \varphi_{2}\right) & \cdots & R\left(1-\cos \varphi_{6}\right) \\ 1 & \cdots & 1 \\ 0 & \cdots & 0 \\ K_{2} & \cdots & 0 \\ \vdots & \vdots & \vdots \\ 0 & \cdots & K_{6}\end{array}\right]^{-1}\left[\begin{array}{l}\sum u_{P}^{L}(2 \pi) \\ \sum v_{P}^{L}(2 \pi) \\ \sum \theta_{P}^{L}(2 \pi) \\ \sum M_{P}^{L}\left(\varphi_{1}\right) \\ \sum M_{P}^{L}\left(\varphi_{2}\right) \\ \vdots \\ \sum M_{P}^{L}\left(\varphi_{6}\right)\end{array}\right]$

As for the examples presented in the sequel, such a trial-and-error approach could be completed in two steps. As for the first step, the stiffnesses of all six interfaces is set equal to the arithmetic mean of $K^{+}$and $K^{-}$:

$K_{1}=K_{2}=\cdots=K_{6}=\frac{K^{+}+K^{-}}{2}$.

After the solution of Eq. (26), the integration constants and the relative rotation angles are known. Inserting them into the transfer relations (1), allows for calculation of the bending moments transmitted at the interfaces. The mathematical signs of these bending moments allows for assigning either $\mathrm{K}^{+}$or $\mathrm{K}^{-}$to each one of the interfaces. These updated values are inserted into Eq. (26). 
The second solution of Eq. (26), based on the updated values of the spring stiffnesses, delivers updated values of the integration constants and the relative rotation angles. Inserting them into the transfer relations (1), allows for calculation of the bending moments transmitted at the interfaces. As for the examples presented in the sequel, the signs of the bending moments did not change from the first to the second solution. This proves that proper values of the spring stiffnesses were used for the second step. The obtained transfer relations allow for computation of the state vector at an arbitrary crosssection of interest simply by multiplying the known transfer matrix with the known vector of kinematic and static variables of the initial cross-section.

\section{STRUCTURAL ANALYSIS OF A REAL- SCALE TEST OF A SEGMENTED TUNNEL RING}

\subsection{Application of the proposed analysis method}

The proposed mode of structural analysis is applied to re-analysis of the initial phase of a real-scale test of a segmented tunnel ring (Liu et al. 2016), see Fig. 1. The structural analysis focuses on the first four load steps, during which the reinforced concrete segments remained uncracked. Therefore, linear elastic behavior of the reinforced concrete segments and a linear interface law appear as reasonable assumptions.

The radius of the tested ring, $R$, is equal to $2.925 \mathrm{~m}$ see Fig. 2(a). The ring is an assembly of six precast reinforced concrete segments, named $K, A$,
$B, C, D, E$. The segment-to-segment interfaces were positioned at angular coordinates $\varphi_{j=1,2, \ldots, 6}=\left[8^{\circ}\right.$, $73^{\circ}, 138^{\circ}, 222^{\circ}, 287^{\circ}, 352^{\circ}$, whereby the angular coordinate $\varphi$ is measured from the crown of the segmented tunnel ring, i.e. from the cross-section in the middle of segment $K$. The thickness and the axial length of the segments amount to $0.35 \mathrm{~m}$ and $1.2 \mathrm{~m}$, respectively. The extensional stiffness $E A$ and the bending stiffness $E I$ of the steel-reinforced concrete segments are given as 18,260 $\mathrm{MN}$ and $186 \mathrm{MNm}^{2}$, respectively. Compressive loading was imposed by 24 equally distributed hydraulic jacks, see Fig. 2(b). The point loads were imposed at angular positions $\varphi_{p=1,2 \ldots 24}=\left[0^{\circ}, 15^{\circ}, 30^{\circ}, \ldots 345^{\circ}\right]$. They simulate the anisotropic ground pressure,

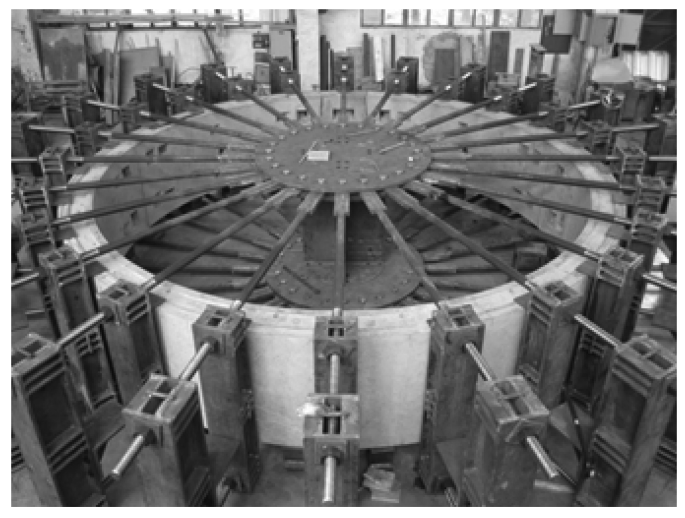

Figure 1. Setup of the analyzed real-scale experiment on a segmented tunnel ring (Liu et al. 2016).

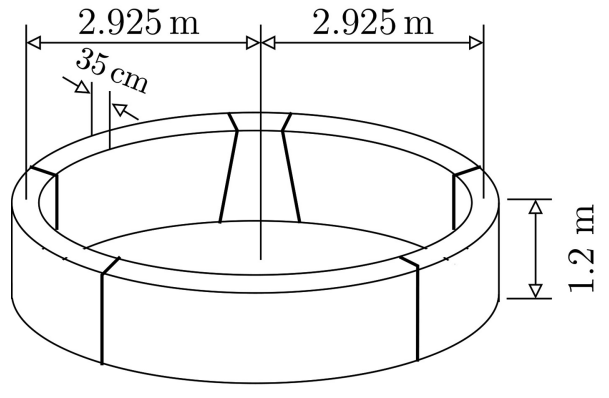

(a)

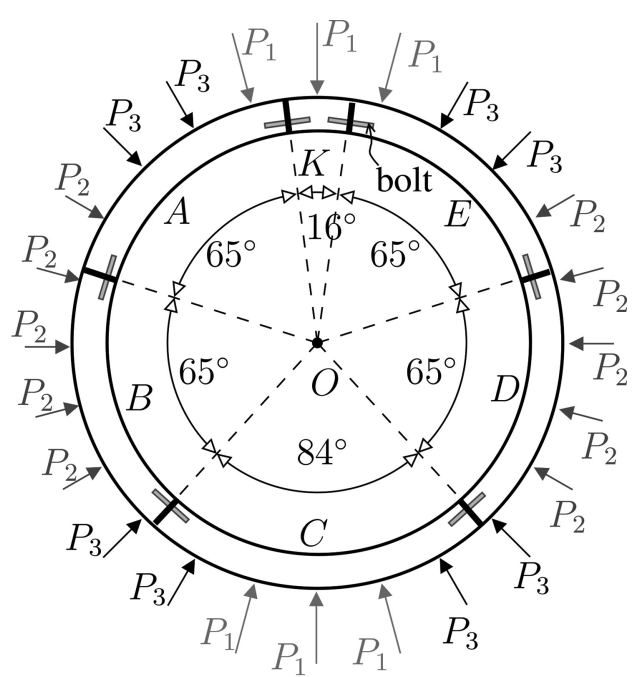

(b)

Figure 2. Geometric dimensions of the analyzed segmented tunnel ring and its external loading: (a) axonometric sketch of the ring, (b) top view of the ring, illustrating the layout of the hydraulic jacks (Zhang et al. 2017). 


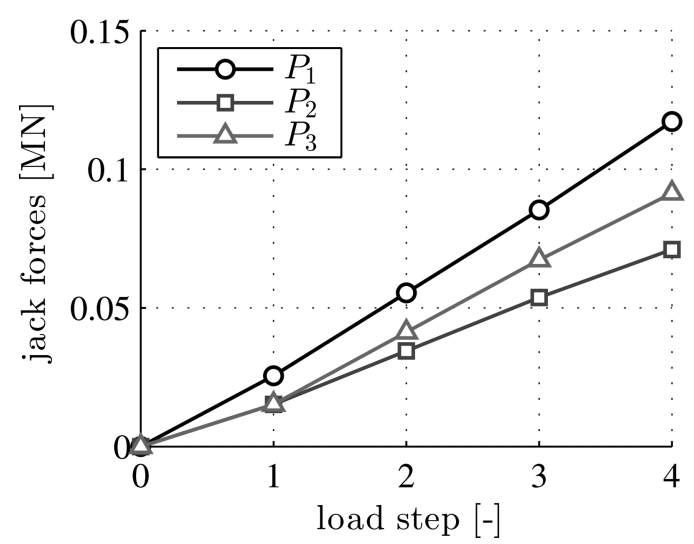

Figure 3. Numerical values of the hydraulic jack forces imposed onto the segmented tunnel ring during the first four load steps of the test (Zhang et al. 2017).

see Fig. 3. The numerical values of the rotational spring stiffnesses of the interfaces are taken from (Liu et al. 2017). They read as

$K^{-}=11.0 \mathrm{MNm} / \mathrm{rad}$,

$K^{+}=6.5 \mathrm{MNm} / \mathrm{rad}$

For each one of the four considered load steps, the solution strategy described in Subsection 2.4 is used. In this context, Eq. (26) is evaluated for (i) the load integrals concerning the point loads, taken from Eqs. (15)-(18), (ii) the numerical input values from Table 1, (iii) the hydraulic jack forces from Fig. 3, and (iv) the assigned spring stiffnesses $K_{1,2 \ldots, 6}$. Table 2 summarizes the obtained numerical results for the three integration constants and the six relative rotation angles. After the two-step solution of Eq. (26), described in Subsection 2.4, the transfer relations (1) are specified (i) for the expressions (2)-(14), (ii) the load integrals (15)(24), (iii) the numerical input values from Table 1 , (iv) the hydraulic jack forces illustrated in Fig. 3, (v) the vanishing kinematic variables at the initial cross-section, see (27), and (vi) the results obtained from the solution of Eq. (26), see Table 2. The obtained transfer relations may be evaluated for any angular position $\varphi$ of interest. The computed kinematic variables allow for illustrating the deformed configuration (Fig. 4), following the formula (Zhang et al. 2017).

$$
\begin{aligned}
\mathbf{u}(\varphi, r) & =u(\varphi) \mathbf{e}_{r}(\varphi) \\
& +[\nu(\varphi)-(r-R) \theta(\varphi)] \mathbf{e}_{\varphi}(\varphi),
\end{aligned}
$$

where $\mathbf{u}, \mathbf{e}_{r}$, and $\mathbf{e}_{\varphi}$ stand for the displacement vector and for the unit base vectors in the radial and
Table 1. Properties of the segmented tunnel ring, occurring in the transfer relations.

\begin{tabular}{ll}
\hline quantity & Numerical value \\
\hline radius of the axis of the ring & $R=2.925 \mathrm{~m}$ \\
bending stiffness & $E I=186 \mathrm{MNm}^{2}$ \\
extensional stiffness & $E A=18,260 \mathrm{MN}$ \\
position of interface 1 & $\varphi_{1}=8^{\circ}$ \\
position of interface 2 & $\varphi_{2}=73^{\circ}$ \\
position of interface 3 & $\varphi_{3}=138^{\circ}$ \\
position of interface 4 & $\varphi_{4}=222^{\circ}$ \\
position of interface 5 & $\varphi_{5}=287^{\circ}$ \\
position of interface 6 & $\varphi_{6}=352^{\circ}$ \\
position of the $k t h$ hydraulic jack & $\varphi_{k}=(k-1) \cdot 15^{\circ}$ \\
\hline
\end{tabular}

Table 2. Numerical values of (i) the internal forces at the crown of the ring and (ii) the relative rotation angles at the interfaces, obtained from the numerical evaluation of Eq. (26).

\begin{tabular}{lcccc}
\hline Loadstep & \multicolumn{1}{l}{1} & \multicolumn{2}{l}{3} & \multicolumn{1}{l}{4} \\
\hline$M_{i}[\mathrm{MNm}]$ & -0.0172 & -0.0383 & -0.0594 & -0.0864 \\
$N_{i}[\mathrm{MN}]$ & -0.0627 & -0.1489 & -0.2351 & -0.3158 \\
$V_{i}[\mathrm{MN}]$ & 0.0128 & 0.0277 & 0.0427 & 0.0586 \\
$\Delta \theta_{1}\left[10^{-3} \mathrm{rad}\right]$ & -1.25 & -2.84 & -4.43 & -6.50 \\
$\Delta \theta_{2}\left[10^{-3} \mathrm{rad}\right]$ & 2.01 & 4.62 & 7.25 & 10.64 \\
$\Delta \theta_{3}\left[10^{-3} \mathrm{rad}\right]$ & -0.64 & -1.53 & -2.42 & -3.56 \\
$\Delta \theta_{4}\left[10^{-3} \mathrm{rad}\right]$ & -0.64 & -1.53 & -2.42 & -3.56 \\
$\Delta \theta_{5}\left[10^{-3} \mathrm{rad}\right]$ & 2.01 & 4.62 & 7.25 & 10.64 \\
$\Delta \theta_{6}\left[10^{-3} \mathrm{rad}\right]$ & -1.25 & -2.84 & -4.43 & -6.50 \\
\hline
\end{tabular}

the circumferential directions, respectively. The distribution of the normal stresses (Fig. 4) follows from (Melan 1913).

$\sigma_{\varphi \varphi}(\varphi, r)=\frac{N(\varphi)}{A}+\frac{M(\varphi)}{I}(r-R)$,

where $A$ and $I$ denote the cross-sectional area and the moment of inertia, respectively.

\subsection{Comparison of results from the presented analysis with results from hybrid analysis of the same structural test}

It is interesting to compare the results from the presented structural analysis with the corresponding results from hybrid analysis of the same test, where both the imposed jack forces and the measured interfacial discontinuities were used as input (Zhang et al. 2017). The results obtained from structural analysis, described in Subsection 3.1, refer to a symmetric answer of the symmetrically loaded segmented tunnel ring. Results from the 


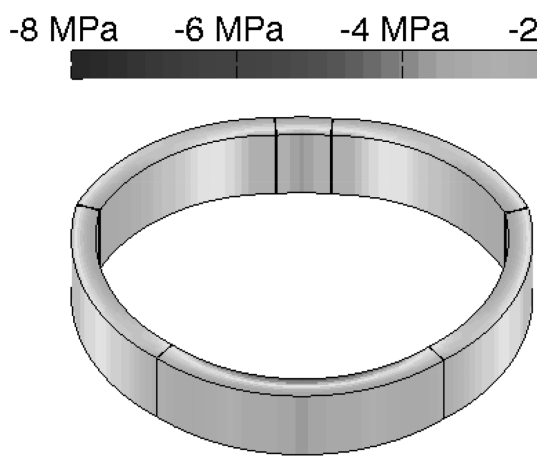

(a)

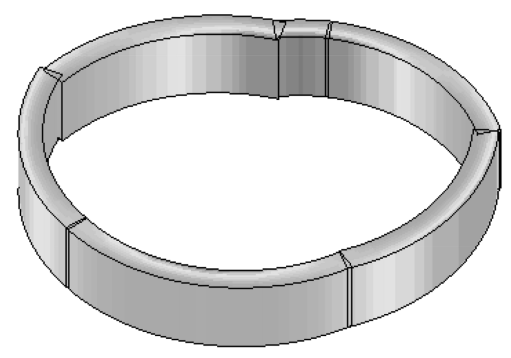

(c)

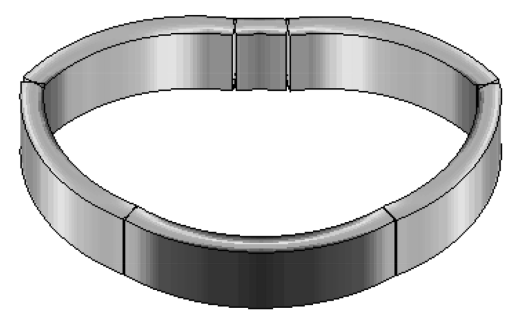

(b)

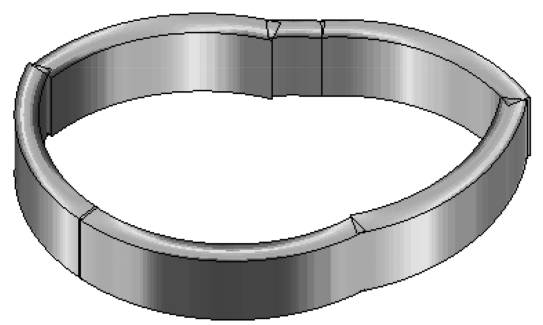

(d)

Figure 4. Deformed configuration of the analyzed segmented tunnel ring and distribution of the normal stresses according to Eqs. (31) and (32): (a) load step 2 and (b) load step 4, analyzed by the presented approach, where the interfacial discontinuities are predicted by the interface model (25); (c) load step 2 and (d) load step 4, analyzed by the hybrid method (Zhang et al. 2017), where the measured interfacial discontinuities were used as input; the magnification factor of the displacements amounts to 50 .

hybrid analysis, in turn, indicate a nonsymmetric answer of the symmetrically loaded segmented tunnel ring.

The differences concerning the inner forces and the corresponding stresses are rather small. The largest differences were obtained for the bending moment, compare Figs. 5(a) and (d). The differences between the normal stresses were insignificant (Fig. 4), because the stress states are dominated by the compressive normal forces.

The computed displacements, in turn, show quite significant differences, compare Figs. 4(a) and (c) as well as Figs. 4(b) and (d). This is because the tested ring exhibited initial geometric imperfections. The latter resulted in nonsymmetric measured interfacial discontinuities (Zhang et al. 2017). Notably, the nonsymmetric part of the measured displacement discontinuities refers mostly to rigid body motions. This explains why the present analysis delivers reliable estimates of the inner forces, but underestimates the displacements.

\section{SUMMARY AND CONCLUSIONS}

A linear interface law, see (25), was combined with transfer relations (1). This allowed for performing design-like structural simulations of a segmented tunnel ring, subjected to known external loading. The conceptual advantage of the presented analysis method over the competing hybrid method, see (Zhang et al. 2017), is that the latter requires measurements of interfacial discontinuities, while the former does not need such measurements.

In the context of the analyzed real-scale test, it was shown that the presented method delivers good estimates of the inner forces. Thus, the interface law (25) accounted reasonably well for the 


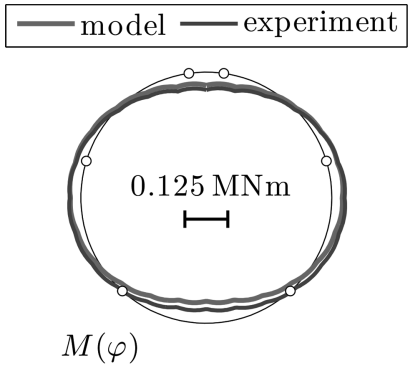

(a)

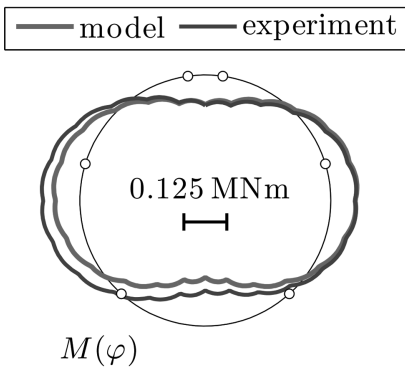

(d)

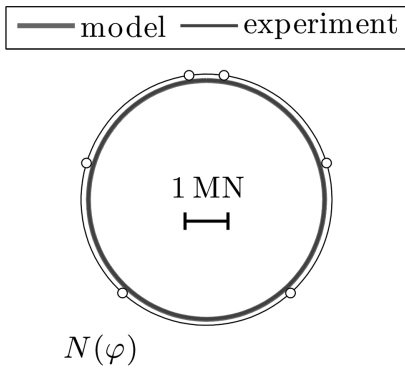

(b)

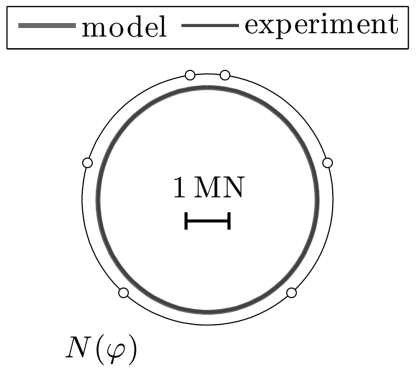

(e)

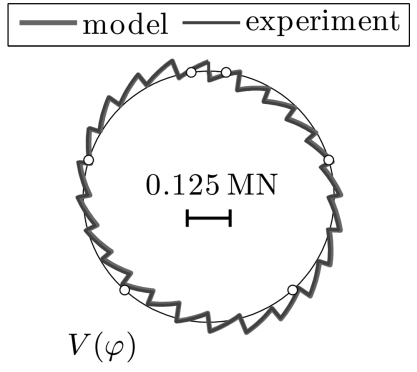

(c)

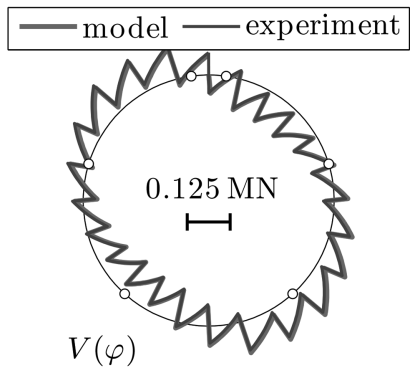

(f)

Figure 5. Distributions of internal forces of the analyzed segmented tunnel ring: (a)-(c) load step 2, (d)-(f) load step 4; the red graphs are obtained from the presented approach, where the interfacial discontinuities are predicted by the interface model (25); the blue graphs are obtained from hybrid analysis (Zhang et al. 2017), where the measured interfacial discontinuities were used as input.

discontinuities induced by the inner forces, transmitted across the interfaces. The displacements of the analyzed tunnel ring, however, were predicted with less accuracy. They consist of two parts, one of which is caused by the inner forces, while the other one is the consequence of discontinuities due to rigid body motions, which are not considered by the proposed interface law.

As for the analysis of Serviceability Limit States, both the inner forces and the displacements must be quantified reliably. For such states, the hybrid method remains the "gold standard", because interface models cannot predict rigid body motions of the segments.

As for the analysis of Ultimate Limit States, a reliable quantification of the inner forces is necessary, while accurate values of the displacements are less important. In this context, it seems that the proposed approach may be useful for engineering practice. Anyhow, this requires the development of (i) transfer relations, capable of accounting for the nonlinear behavior of reinforced concrete segments, and of (ii) nonlinear interface laws. The latter should be supported by real-scale tests on socalled "concrete hinges" (Schlappal et al. 2017) and corresponding numerical re-analyses, based on the Finite Element method (Kalliauer et al. 2017).

\section{ACKNOWLEDGMENT}

Financial support by the Austrian Science Fund (FWF), provided within project P 281 31-N32 "Bridging the Gap by Means of Multiscale Structural Analyses", and interesting discussions with Thomas Schlappal (TU Wien) are gratefully acknowledged. In addition, the first author gratefully acknowledges financial support by the China Scholarship Council.

\section{REFERENCES}

Brandtner, M., B. Moritz, \& P. Schubert (2007). On the challenge of evaluating stress in a shotcrete lining: 
Experiences gained in applying the hybrid analysis method. Felsbau 25(5), 93-98.

Gladwell, G.M.L. (1980, June). Contact problems in the classical theory of elasticity. Springer Science \& Business Media.

Hellmich, C. (1999). Shotcrete as part of the new Austrian tunneling method: From thermochemomechanical material modeling to structural analysis and safety assessment of tunnels. Ph. D. thesis, Vienna University of Technology, Vienna, Austria.

Hellmich, C., J. Macht, \& H.A. Mang (1999). Ein hybrids Verfahren zur Bestimmung der Auslastung von Spritzbetonschalen [A hybrid method for determination of the degree of utilization of shotcrete tunnel shells]. Felsbau 17(5), 422-425. In German.

Hellmich, C., H.A. Mang, \& F.-J. Ulm (2001). Hybrid method for quantification of stress states in shotcrete tunnel shells: combination of $3 \mathrm{D}$ in situ displacement measurements and thermochemoplastic material law. Computers \& Structures 79(22), 2103-2115.

Janßen, P. (1983, December). Tragverhalten von Tunnelausbauten mit Gelenkstübbings [Structural behavior of segmented tunnel linings $7 . \mathrm{Ph}$. D. thesis, Technical University of Braunschweig. In German.

Kalliauer, J., T. Schlappal, M. Vill, H.A. Mang, \& B. Pichler (2017). Bearing capacity of concrete hinges subjected to eccentric compression: Multiscale structural analysis of experiments. Acta Mechanica. accepted for publication.

Leonhardt, F. \& H. Reimann (1965). Betongelenke: Versuchsbericht, Vorschläge zur Bemessung und konstruktiven Ausbildung. Kritische Spannungszustände des Betons bei mehrachsiger, ruhender Kurzzeitbelastung [Concrete hinges: test report, recommendations for structural design. Critical stress states of concrete under multiaxial static short-term loading], Volume 175. Ernst und Sohn. In German.

Liu, X., Y. Bai, Y. Yuan, \& H.A. Mang (2016). Experimental investigation of the ultimate bearing capacity of continuously jointed segmental tunnel linings. Structure and Infrastructure Engineering 12(10), 1364-1379.

Liu, X., C. Zhang, C. Zhang, \& Y. Yuan (2017). Ultimate loadcarrying capacity of the longitudinal joints in segmental tunnel linings: Illustrated with the typi- cal segment joints of the Shanghai Metro rapid transit system. Structural Concrete 18(5), 693-709. 10.1002/ suco. 201600070 .

Melan, J. (1913). Theory of arches and suspension bridges. Chicago, Clark.

Pichler, B., S. Scheiner, \& C. Hellmich (2008). From micronsized needle-shaped hydrates to meter-sized shotcrete tunnel shells: Micromechanical upscaling of stiffness and strength of hydrating shotcrete. Acta Geotechnica 3(4), 273-294.

Rokahr, R. \& R. Zachow (1997). Ein neues Verfahren zur täglichen Kontrolle der Auslastung einer Spritzbetonschale [A new method for the daily monitoring of the stress intensity of a sprayed concrete lining]. Felsbau 15(6), 430-434. In German.

Rubin, H. \& U. Vogel (1993). Baustatik ebener Stabwerke [Frameworks for structural analysis]. Köln: Stahlbau Handbuch Für Studium und Praxis BAND 1 Teil A, Stahlbau-Verlagsgesellschaft mbH. In German.

Schlappal, T., M. Schweigler, S. Gmainer, M. Peyerl, \& B. Pichler (2017). Creep and cracking of concrete hinges-Insight from centric and eccentric compression experiments. Materials and Structures. accepted for publication.

Ullah, S., B. Pichler, \& C. Hellmich (2013). Modeling ground-shell contact forces in NATM tunneling based on three-dimensional displacement measurements. Journal of Geotechnical and Geoenvironmental Engineering 139(3), 444-457.

Ullah, S., B. Pichler, S. Scheiner, \& C. Hellmich (2010). Shellspecific interpolation of measured 3D displacements, for micromechanics-based rapid safety assessment of shotcrete tunnels. Computer Modeling in Engineering and Sciences (CMES) 57(3), 279-315.

Ullah, S., B. Pichler, S. Scheiner, \& C. Hellmich (2012). Influence of shotcrete composition on load-level estimation in NATM-tunnel shells: Micromechanicsbased sensitivity analyses. International Journal for Numerical and Analytical Methods in Geomechanics 36(9), 1151-1180.

Zhang, J.-L., C. Vida, Y. Yuan, C. Hellmich, H.A. Mang, \& B. Pichler (2017). A hybrid analysis method for displacement-monitored segmented circular tunnel rings. Engineering Structures 148, 839-856. 